\title{
APLICAÇÃO DO MÉTODO THOR PARA ESCOLHA DE UM MODELO DE TREINAMENTO EM UM LABORATÓRIO DE METROLOGIA
}

\author{
Ana Lívia Caitano \\ Universidade Unigranrio \\ Rua Prof. José de Souza Herdy, 1.160 - Jardim 25 de Agosto, Duque de Caxias/RJ \\ livia.alvescaitano@gmail.com
}

Marcos dos Santos

Centro de Análises de Sistemas Navais / Instituto Militar de Engenharia

Praça Barão de Ladário s/n, Ilha das Cobras, Rua da Ponte, Ed. 23 do AMRJ, Centro, Rio de Janeiro/RJ

Praça General Tibúrcio, nº 80, Praia Vermelha, Urca, Rio de Janeiro/RJ

marcosdossantos_doutorado_uff@yahoo.com.br

Carlos Francisco Simões Gomes

Universidade Federal Fluminense

Rua Passo da Pátria, nº 156, Escola de Engenharia, São Domingos, Niterói/RJ

cfsg1@bol.com.br

Fabricio Maione Tenório

Instituto Militar de Engenharia / Centro Federal de Educação Tecnológica Celso Suckow da Fonseca

Praça General Tibúrcio, nº 80, Praia Vermelha, Urca, Rio de Janeiro/RJ

Rodovia Mário Covas, lote J2, quadra J, Distrito Industrial de Itaguaí, Itaguaí/RJ

fabriciomte@gmail.com

\section{RESUMO}

O treinamento e o desenvolvimento desempenham um papel importante na eficácia e eficiência das organizações, bem como nas experiências dos indivíduos no trabalho. Foi utilizado a metodologia multicritério para sugerir a escolha de um novo modelo de treinamento. Os modelos são: 4 níveis de Donald Kirkpatrick, modelo do valor final de Hamblin, modelo MAIS de Borges-Andrade, modelo IMPACT de ABBAD e modelo IMTEE. O estudo é aplicado em um laboratório de metrologia, pois os procedimentos tradicionais utilizados nos sistemas de avaliação estão sendo questionados quanto à possibilidade de acompanharem adequadamente e oferecerem respostas à contribuição das ações de treinamento e desenvolvimento para a organização. O Método THOR foi desenvolvido em 1999 e é aplicado em problemas de ordenação de alternativas; podendo ser utilizado em situações nas quais há ausência de um dado na matriz de decisão sem a necessidade de eliminar uma alternativa ou critério. Este método mostrou-se uma ferramenta adequada para balizar os processos de tomada de decisão, sendo possível chegar a um resultado satisfatório. Após aplicação do método, chegou-se à conclusão de que o modelo "MAIS" foi escolhido como o modelo padrão que irá nortear o desenvolvimento das atividades de treinamento no laboratório.

Palavra-chave: Apoio Multicritério à Decisão; Treinamento; THOR. 


\begin{abstract}
Training and development play an important role in the effectiveness of organizations and in the experiences of individuals at work. The multicriteria methodology was used to choose a new training model, between them, the Donald Kirkpatrick 4-level model, Hamblin final value model, Borges-Andrade MAIS model, ABBAD IMPACT model and the IMTEE model, to apply in a metrology laboratory, because traditional procedures used in assessment systems are being questioned if they can adequately follow up and provide answers to the contribution of training and development actions to the organization. The THOR Method was developed in 1999 and solves alternative ordering problems in situations where data is missing from the decision matrix without the need to eliminate an alternative or criterion. This method proved to be an adequate tool to guide decision making processes, making it possible to reach an objective result. After applying the method, it was concluded that the "MAIS" model was chosen as the standard model that will guide the development of training activities in the laboratory.
\end{abstract}

Keywords: Multi-criteria decision analysis; Training; THOR

\title{
Como Citar:
}

CAITANO, Ana Lívia; SANTOS, Marcos dos; GOMES, Carlos Francisco Simões; TENORIO, Fabricio Maione. Método THOR para escolha de um modelo de treinamento em laboratório. In: SIMPÓSIO DE PESQUISA OPERACIONAL E LOGÍSTICA DA MARINHA, 19., 2019, Rio de Janeiro, RJ. Anais [...]. Rio de Janeiro: Centro de Análises de Sistemas Navais, 2019.

\section{INTRODUÇÃO}

Com o objetivo de capacitar seus colaboradores e, consequentemente, elevar sua produtividade, as organizações estão investindo cada vez mais em treinamentos. O investimento em treinamento e desenvolvimento (T\&D) em 2017 foi, em média, R\$ 788,00 por colaborador. $\mathrm{Na}$ área da indústria e serviços, o valor se eleva para cerca de $\mathrm{R} \$ 820,00$, resultando em um crescimento de $21 \%$ em relação ao ano anterior (cerca de $\mathrm{R} \$ 622,52$ ), um valor ainda muito aquém do investimento em T\&D das empresas dos EUA (cerca de U\$ $1.252,00$, ou $\mathrm{R} \$ 4.820,00$ por colaborador). Este indicador vem crescendo ano a ano, apontando, sem dúvida, uma constante tendência de ganho de relevância [1].

Em um total de 738 empresas avaliadas, verificou-se que: $79 \%$ dos treinamentos limitam-se apenas à avaliação de reação, nas quais o aluno avalia a qualidade e aplicabilidade do curso; $28 \%$ das ações de treinamento têm avaliação de aprendizado, na qual verifica-se o ganho de conhecimento por parte do aluno; $12 \%$ dos projetos avaliam se o profissional aplicou os conhecimentos adquiridos; $5 \%$ avaliam qual o impacto no resultado do negócio; e $2 \%$ avaliam o retorno financeiro sobre o investimento [1].

A falta de indicadores adequados para mensurar os resultados dos investimentos em treinamentos, geram, por sua vez, ceticismo em parte dos gestores a respeito da necessidade de um maior investimento em T\&D.

Segundo o relatório da ABTD [1], pouco se mudou nos últimos anos sobre a escolha dos indicadores de avaliação de treinamento, sendo o maior deles a satisfação do colaborador com o trabalho, um indicador muito subjetivo. 
O presente estudo tem como objetivo auxiliar a escolha de um modelo de treinamento adequado ao contexto de um laboratório de Metrologia de pequeno porte situado no Rio de Janeiro. Para tal, será utilizado o método multicritério de apoio à decisão THOR.

\section{DESCRIÇÃO DO PROBLEMA}

Os procedimentos tradicionais utilizados nos sistemas de avaliação estão sendo questionados quanto à possibilidade de acompanharem adequadamente e oferecerem respostas à contribuição das ações de treinamento e desenvolvimento para a organização. Novelli [2] descreve algumas razões para esse questionamento, por se observar um enfoque didáticopedagógico direcionado à avaliação da metodologia, conteúdo e recursos do treinamento, sem dar a devida importância a sua contribuição para o alcance dos objetivos estratégicos das organizações.

Apesar do crescente interesse dos pesquisadores sobre os métodos avaliativos de treinamentos, medidas válidas e confiáveis de satisfação com o treinamento são ainda raras. A literatura especializada em $T \& D$ vem mostrando resultados inconsistentes no que tange ao relacionamento de variáveis de reações ao treinamento com medidas tradicionais como aprendizagem e comportamento no cargo, em parte devido à falta de sistemáticas objetivas para essa avaliação [3].

Em paralelo a isso, para um laboratório de metrologia, a acreditação segundo os requisitos da ISO 17025:2017 [4], que trata dos requisitos gerais para a competência de laboratórios de ensaio e calibração, é importantíssima para o seu sucesso, pois permite ao laboratório se colocar estrategicamente no mercado, sendo reconhecido por seus clientes como um laboratório de excelência e reconhecida competência.

A ISO 17.025:2017 [4] determina que os laboratórios devem possuir e prover pessoas capacitadas para a realização das suas atividades de forma adequada, levando a pergunta fundamental a ser respondida por este trabalho ter como formulação: "É possível com o apoio da metodologia multicritério de apoio à decisão auxiliar no processo de escolha de um modelo para a avaliação da eficácia do treinamento de novos funcionários mais adequado ao ambiente de um laboratório de metrologia com acreditação?".

Apesar do mercado ofertar capacitações em âmbito geral, há uma significativa lacuna entre a teoria destes treinamentos e a prática no cotidiano de um laboratório. Como solução, as empresas estão investindo na capacitação interna. O treinamento interno é uma ferramenta indispensável para que ocorram ganhos de conhecimento e habilidades, permitindo reduzir custos e tempo no processo de capacitação de equipes.

No entanto, na literatura quase não existem metodologias para a avaliação desses treinamentos, resultando em funcionários não capacitados adequadamente para exercer suas funções, gerando como consequência: erros de resultados, retrabalho, não conformidades e acidentes de trabalho. Este risco, por si só, justifica o estudo desenvolvido nesse trabalho.

O estudo de caso foi realizado em um laboratório de metrologia situado na cidade do Rio de Janeiro, e foi escolhido devido à facilidade de um dos autores às informações e aos entrevistados. Para Gil [5], o estudo de caso é caracterizado pelo estudo profundo e exaustivo de um ou de poucos objetos, de maneira a permitir o seu conhecimento amplo e detalhado.

\section{FUNDAMENTAÇÃO TEÓRICA}

Loureiro [6] afirma que o treinamento é a atividade responsável dedicada à transmissão de conhecimentos objetivando suprir deficiências, estimular e desenvolver habilidades e potencialidades, visando um crescimento tanto no aspecto profissional/cultural 
do indivíduo, quanto da empresa, no que se refere à obtenção e manutenção de mão-de-obra mais qualificada e preparada para assimilar e superar desafios.

\subsection{Treinamento e Desenvolvimento}

Para Foltran [7], o treinamento é um processo cíclico que envolve as seguintes etapas: levantamento das necessidades específicas dos treinamentos, aplicação dos princípios de aprendizagem, escolhas de métodos adequados à instrução, coleta de dados e materiais para a instrução, elaboração do programa de treinamento, treinamento dos instrutores, execução dos treinamentos, avaliação e controle dos resultados dos treinamentos. Borges-Andrade [8] delimita o treinamento como um sistema composto por três subsistemas: levantamento de necessidades, planejamento e execução e avaliação do treinamento. O subsistema avaliação, em especial, fornece a retroalimentação para todo o sistema, indicando os pontos positivos que devem permanecer nos treinamentos futuros, bem como sinalizando aspectos a serem modificados ou retirados do planejamento. Chiavenato [9] define o treinamento como sendo o processo educacional de curto prazo, aplicado de maneira sistemática e organizada. Diante disto, como instrumento de intervenção, foi utilizada a metodologia multicritério de apoio à decisão por se tratar de uma ferramenta que permite construir conhecimento do contexto de um problema com múltiplas alternativas, hierarquizando-as de acordo com critérios estabelecidos, auxiliando o decisor para a tomada de decisão.

Para Borges-Andrade [10], existe uma demanda cada vez maior por avaliação de treinamento nas organizações, que tradicionalmente fizeram altos investimentos em treinamento, bem como naquelas que mais recentemente descobriram os valores estratégicos do conhecimento e do esforço na contínua qualificação de seu pessoal.

As instituições que contratam esse tipo de serviço estão interessadas em garantir a aplicação dos saberes que o participante adquiriu, preferencialmente de maneira imediata, em sua atividade profissional e quanto desse aprendizado pode se manter ao longo do tempo [11].

Santos et al. [12] afirmam que a Pesquisa Operacional (PO) lança mão de modelos matemáticos e/ou lógicos, a fim de resolver problemas reais, apresentando um caráter eminentemente multidisciplinar.

Abbad et al. [13] classifica os Modelos de Avaliação de Treinamento Desenvolvimento e Educação (TD\&E) em:

1) genéricos - baseados em combinações de variáveis relacionadas aos processos de TD\&E, tais como o modelo clássico de Kirkpatrick [14], aperfeiçoado por Hamblin [15] e o Modelo de Avaliação Integrado e Somativo (MAIS) [16].

2) específicos - baseados em modelos genéricos, são formados a fim de testar a relação entre associações de variáveis e resultados de TD\&E a nível individual, no grupo de trabalho, na organização ou extra organizacional. Os que mais se destacam são os modelos: Modelo Integrado de Avaliação do Impacto do Treinamento no Trabalho (IMPACT) [17].

Apesar de muito importantes, medidas válidas e confiáveis de satisfação com o treinamento são ainda raras. Destacam-se, sobretudo, os trabalhos de Kirkpatrick [14], Hamblin [15], Borges-Andrade [16], Abbad [17] e Alvarez et al. [18].

\subsection{Modelos de Treinamento}

Segue a descrição dos modelos a serem avaliados no estudo. 


\subsubsection{Modelo dos 4 níveis}

Um modelo clássico é o sugerido por Kirkpatrick [14], que define quatro níveis de avaliação: reações, aprendizagem, comportamento no cargo e resultados.

As reações representam medidas de satisfação com o treinamento em termos de qualidade do programa, instalações e desempenho do instrutor. A aprendizagem diz respeito à avaliação das diferenças de desempenho dos treinandos antes e depois do treinamento, em termos dos objetivos instrucionais. O comportamento no cargo aborda as melhorias no desempenho do treinando no trabalho, decorrentes da aplicação do que foi aprendido no treinamento. Os resultados enfocam as mudanças ocorridas no nível da organização em função do treinamento.

\subsubsection{Modelo do Valor Final}

Hamblin [15] propôs uma avaliação de treinamento a partir das pesquisas de Kirkpatrick [14] e incluiu um quinto nível, o valor final, que tem como foco a produção ou o serviço prestado pela organização, o que geralmente implica em comparar custos do treinamento com os seus benefícios.

Se a finalidade da ação é provocar um aprendizado que deve se refletir em mudança de comportamento, justifica-se o foco da avaliação do resultado como mudança para a organização e valor definitivo do treinamento.

\subsubsection{Modelo MAIS}

No Brasil, Borges-Andrade [16] foi um dos primeiros autores nacionais responsáveis pela criação de modelos de avaliação de treinamento e desenvolvimento considerando outras variáveis que não as relativas puramente aos resultados do treinamento.

Borges-Andrade [16] propôs um modelo que inclui variáveis do ambiente como fatores influentes importantes em todo o processo de treinamento, desde o levantamento de necessidades até os resultados a longo prazo; conhecido como Modelo de Avaliação Integrado e Somativo (MAIS).

O modelo MAIS é composto essencialmente por cinco componentes: insumos, procedimentos, processo, resultados e ambiente; subdividindo-se o Ambiente em: avaliação de necessidades, disseminação, apoio e resultados a longo prazo. Este modelo tem servido de base para muitas intervenções atuais, aplicadas e de pesquisa, em diversos contextos organizacionais.

\subsubsection{Modelo IMPACT}

O Modelo Integrado de Avaliação do Impacto do Treinamento (IMPACT), criado por Abbad [17] para avaliação de treinamento é composto por sete componentes: percepção de suporte organizacional, características do treinamento, características da clientela, reação, aprendizagem, suporte à transferência e impacto do treinamento no trabalho. Em breve detalhamento, a Percepção de Suporte Organizacional se apresenta como elemento multidimensional. Nesse aspecto, encontram-se a opinião que os participantes têm sobre as práticas organizacionais de gestão de desempenho, a valorização do funcionário e o apoio gerencial ao treinamento. A Percepção de Suporte Organizacional parece investigar o apoio psicossocial com o qual o treinando conta, ou julga contar, no contexto de realização do treinamento. 


\subsubsection{Modelo IMTEE}

Alvarez et al. [18] construíram o Modelo Integrado de Avaliação e Efetividade de Treinamento (IMTEE). A construção do IMTEE foi baseada numa revisão de literatura no período de 1993 a 2002.

No estudo, os autores definem com clareza as diferenças entre avaliação e efetividade

de treinamento e fornecem aos especialistas valiosa ferramenta que identifica os diversos inter-relacionamentos existentes, e nem sempre visíveis, entre variáveis e características de um programa de treinamento. O IMTEE mensura quatro níveis de avaliação: análise de necessidades, subdividida em conteúdo e projeto do treinamento, mudanças nos treinandos e demandas organizacionais; reações; transferência; e resultados.

A análise do modelo permite verificar que alguns níveis se inter-relacionam formando uma cadeia e constroem um sistema, no qual os resultados dos diversos níveis dependem uns dos outros. De acordo com o IMTEE, os resultados do nível reação dependeriam apenas das características individuais dos treinandos. As mudanças nos treinandos, auto eficácia póstreinamento, aprendizagem cognitiva e desempenho têm como possíveis preditores as características individuais e características do treinamento. O modelo também relata a influência de características individuais, do treinamento e organizacionais sobre a transferência do treinamento e os resultados.

\subsection{Apoio Multicritério à Decisão (AMD)}

O AMD concede a priorização de alternativas em uma situação de critérios conflitantes, buscando satisfazer as restrições, com objetivos conflitantes, ou seja, uma solução de compromisso [19]. Assim sendo, o AMD pode fornecer métodos para o apoio à negociação e/ou decisão em grupo. Segundo Matsatsinis e Samaras [20], o uso do AMD consiste em:

a) estruturar o processo da decisão, identificando regras de decisão, critérios e pesos dos critérios;

b) representar as múltiplas visões dos atores da decisão; e

c) grupar preferências eliciando os valores do grupo.

De acordo com Lyrio [21], a origem do AMD está ligada à área do conhecimento que se denomina PO. Esta área do conhecimento se desenvolveu a partir da segunda guerra mundial, quando os aliados se viram frente a problemas ligados à logística, tática e estratégia militar, buscando nos pesquisadores, principalmente das áreas de matemática, física e engenharia, o embasamento científico para solução deste tipo de problemas, com vistas a desenvolver modelos matemáticos, baseados em dados e fatos, que permitissem analisar o resultado hipotético de estratégias ou decisões alternativas.

Santos et al. [22] afirmam que a PO lança mão de modelos matemáticos e/ou lógicos, a fim de resolver problemas reais, apresentando um caráter eminentemente multidisciplinar.

Os AMD se fundamentam em um conjunto de matrizes ou modelos que irão auxiliar o processo de decisão (individual ou conjunta) de um problema a ser solucionado no qual os critérios são conflitantes [23]. Estas metodologias funcionam como uma base para discussão, principalmente nos casos onde há conflitos entre os decisores, ou ainda, quando a percepção do problema pelos vários atores envolvidos ainda não está totalmente consolidada [24].

Para Vincke [25], uma das grandes vantagens em se utilizar métodos multicritério e pesquisa operacional ocorre pelo fato de que não há, em sua grande maioria, decisões que sejam conjuntamente ótimas sob todos os pontos de análise, fazendo com que ocorra desta forma, a seleção da melhor opção possível. A diferença entre os métodos multicritério de 
tomada de decisão e outros métodos é considerarem diversos conceitos e avaliarem as ações por meio de um conjunto de critérios, derivando de cada conjunto uma função matemática que serve para medir o desempenho dessas ações.

\subsection{Método THOR}

O Método THOR consiste em três algoritmos para uso simultâneo: Modelagem de Preferência (aproximando-o da Escola Francesa), Teoria da Utilidade e Teoria de Multiatributo (aproximando-o da Escola Americana). A aplicação simultânea destas teorias, possibilita que a atratividade de uma alternativa seja quantificada, pela formação de uma função agregação não-transitiva.

De acordo com Gomes et.al [26], o THOR é uma metodologia de ordenação de alternativas discretas que elimina critérios redundantes considerando simultaneamente se a informação é dúbia e se ocorre elevação da imprecisão do processo de decisão (Teoria dos Conjuntos Nebulosos), bem como utiliza a quantificação da imprecisão mediante um processo de decisão AMD.

Conforme Xavier et.al [27], o THOR trata-se de um algoritmo que permite a entrada simultânea de dados em mais de um tomador de decisão, facilitando a expressão de julgamentos em escalas de razão, intervalo ou ordinal.

Os seguintes elementos adicionais podem ser necessários para a aplicação do THOR: (i) um peso para cada critério, representando a importância relativa entre eles; (ii) um limiar de preferência (p) e outro para indiferença (q) para cada critério; (iii) uma definição do domínio da discordância; (iv) caracterização da pertinência dos valores dos pesos atribuídos ao critério; (v) a pertinência da classificação da alternativa no critério. Deve-se ressaltar que as relações alcançadas por meio do THOR têm um quantitativo numérico que representa o "valor da alternativa". Isso é realizado por meio de uma função de valor aditivo. A relação de dominância e a hierarquia dos valores das alternativas são assim construídas. Três situações são admitidas para uma alternativa ser melhor que outra [28].

Uma comparação das sugestões de classificação geradas pelo THOR fornece as seguintes vantagens:

1) É possível quantificar as incertezas no processo de priorização;

2) Sugerir a eliminação de critérios que não impactam o processo de classificação;

3) Pode-se aplicar a decisões transitivas e não transitivas;

4) É mais fácil de operar e gera soluções mais rápidas.

O quantitativo numérico que expressa o "valor da alternativa" através de uma função aditiva pode ser observado nas equações 1,2 e 3 . 


$$
\begin{aligned}
S 1: & \sum_{j=1}^{n}\left(w_{j} \mid a P_{j} b\right)>\sum_{j=1}^{n}\left(w_{j} \mid a Q_{j} b+a I_{j} b+a R_{j} b+b Q_{j} a+b P_{j} a\right) \\
S 2: & \sum_{j=1}^{n}\left(w_{j} \mid a P_{j} b+a Q_{j} b\right)>\sum_{j=1}^{n}\left(w_{j} \mid a I_{j} b+a R_{j} b+b Q_{j} a+b P_{j} a\right) \\
S 3: & \sum_{j=1}^{n}\left(w_{j} \mid a P_{j} b+a Q_{j} b+a I_{j} b\right)>\sum_{j=1}^{n}\left(w_{j} \mid a R_{j} b+b Q_{j} a+b P_{j} a\right)
\end{aligned}
$$

Ressalta-se que as relações de sobreclassificação possuem um quantitativo numérico que representam o valor da alternativa, mediante o uso de uma função de valor aditiva [29].

Essa aproximação permite representar a relação de sobreclassificação e a hierarquia dos valores das alternativas. Para que uma alternativa seja melhor do que a outra, três situações devem ser admitidas [30].

Para Tenório et.al [31], dadas duas alternativas $a$ e $b$, três situações devem ser consideradas na utilização do THOR: S1, S2 e S3. Na utilização do algoritmo S1, as alternativas só têm sua atratividade pontuada em situações onde ocorre aPjb. Desta forma, comparando a alternativa a com as demais alternativas, podemos identificar os critérios em que aPjb, levando em conta os limites de preferência ( $\mathrm{P}$ designa preferência estrita, $\mathrm{Q}$ designa preferência fraca), indiferença (I designa indiferença) e discordância, verificando se a condição imposta é satisfeita. Se for satisfeita, sabemos que a domina b. As relações estão evidenciadas nas equações 4,5 e 6 .

$$
\begin{gathered}
a P b \leftrightarrow g(a)-g(b)>p \\
a I b \leftrightarrow-q \leq|g(a)-g(b)| \leq q \\
a Q b \leftrightarrow q<|g(a)-g(b)| \leq p
\end{gathered}
$$

\section{PROPOSTA DE SOLUÇÃO}

\subsection{Levantamento de Dados}

Com a finalidade de auxiliar na escolha do modelo de treinamento, foram levantadas informações para poder traçar o perfil da situação atual do laboratório, mediante a análise dos procedimentos e políticas do local, relacionados a treinamento e capacitação. Em seguida, foram definidos os atores relacionados ao processo, identificando-os de acordo com a classificação da metodologia multicritério de apoio à decisão. Foram definidos em:

(i) Decisores: Diretores, Gerente da Qualidade e Gerente Técnico, Supervisor da divisão de Metrologia Térmica e Supervisor da divisão de Metrologia Físico-química.

(ii) Facilitadores: Autores, também denominada "equipe de implementação"

(iii) Agidos: Funcionários envolvidos nos procedimentos técnicos e de gestão 
Definidos os atores envolvidos no processo e as informações que permitiam uma melhor compreensão do contexto, foi possível avançar na identificação do melhor modelo de treinamento para a realidade do laboratório.

Os modelos de treinamento em estudo foram escolhidos de acordo com sua relevância com o tema e de acordo com a consolidação desses modelos na literatura atual. Os modelos escolhidos foram: Modelo dos 4 níveis [14], Modelo do Valor Final [15], Modelo MAIS [16], Modelo IMPACT [17] e Modelo IMTE [18].

Vale ressaltar que estes modelos foram recebendo revisões de seus próprios autores e de outros pesquisadores, como por exemplo o Modelo dos 4 Níveis onde Kirkpatrick fez revisões até 1993.

A próxima etapa se deu pela elaboração dos critérios e, em seguida, pela aplicação do Método THOR para obtenção do resultado. A descrição dos critérios encontra-se na Tabela 1:

\begin{tabular}{|l|l|}
\hline \multicolumn{1}{|c|}{ Critérios } & \multicolumn{1}{c|}{ Descrição } \\
\hline $\begin{array}{l}\text { Levantamento das } \\
\text { necessidades }\end{array}$ & $\begin{array}{l}\text { Grau em que o modelo de treinamento considera relevante a necessidade } \\
\text { de se realizar o treinamento no laboratório }\end{array}$ \\
\hline Ambiente de Suporte & $\begin{array}{l}\text { Grau de importância e especificação que o modelo de treinamento } \\
\text { considera que o ambiente organizacional deve contribuir na eficácia do } \\
\text { treinamento }\end{array}$ \\
\hline $\begin{array}{l}\text { Complexidade de } \\
\text { Implementação }\end{array}$ & $\begin{array}{l}\text { Grau de complexidade de implementação do modelo de treinamento, } \\
\text { considerando quantidade de etapas, setores que precisam ser envolvidos e } \\
\text { tempo necessário para implementação }\end{array}$ \\
\hline Avaliação das Reações & $\begin{array}{l}\text { Grau de importância e nível de detalhamento em que o modelo de } \\
\text { treinamento avalia as reações dos treinandos e sua influência na eficácia } \\
\text { do treinamento }\end{array}$ \\
\hline $\begin{array}{l}\text { Impacto do } \\
\text { treinamento no } \\
\text { trabalho }\end{array}$ & $\begin{array}{l}\text { Grau e nível de detalhamento em que o modelo de treinamento aborda a } \\
\text { transferência dos ensinamentos aprendidos no treinamento no } \\
\text { comportamento do treinando }\end{array}$ \\
\hline Resultado & $\begin{array}{l}\text { Nível de detalhamento em que o modelo de treinamento considera os } \\
\text { resultados gerados na conclusão do treinamento, se este atendeu às } \\
\text { expectativas iniciais }\end{array}$ \\
\hline
\end{tabular}

Tabela 1: Descrição dos critérios aplicados

\subsection{Modelagem Matemática - Aplicação do Método THOR}

A Tabela 2 apresenta uma matriz com a avaliação das cinco alternativas à luz do conjunto de critérios.

\begin{tabular}{|l|c|c|c|c|c|c|}
\hline & $\begin{array}{c}\text { Levantamento } \\
\text { das } \\
\text { necessidades }\end{array}$ & $\begin{array}{c}\text { Ambiente } \\
\text { de } \\
\text { Suporte }\end{array}$ & $\begin{array}{c}\text { Complexidade } \\
\text { de } \\
\text { implementação }\end{array}$ & $\begin{array}{c}\text { Avaliação } \\
\text { das } \\
\text { reações }\end{array}$ & $\begin{array}{c}\text { Impacto do } \\
\text { treinamento } \\
\text { no trabalho }\end{array}$ & Resultado \\
\hline KIRKPATRICK & 6 & 7 & -30 & 6 & 6 & 11 \\
\hline HAMBLIN & 9 & 17 & -30 & 12 & 8 & 13 \\
\hline IMPACT & 25 & 24 & -12 & 18 & 30 & 25 \\
\hline MAIS & 30 & 30 & -9 & 24 & 25 & 30 \\
\hline IMTEE & 28 & 24 & -12 & 19 & 23 & 19 \\
\hline
\end{tabular}

Tabela 2: Matriz de decisão 
A Tabela 3 apresenta o peso utilizado em cada critério.

\begin{tabular}{|l|c|}
\hline \multicolumn{1}{|c|}{ Critério } & Peso do Critério \\
\hline Levantamento das necessidades & 10 \\
\hline Ambiente de Suporte & 20 \\
\hline Complexidade de implementação & 15 \\
\hline Avaliação das reações & 10 \\
\hline Impacto do treinamento no trabalho & 15 \\
\hline Resultado & 30 \\
\hline
\end{tabular}

Tabela 3: Peso do Critério

A Tabela 4 representa os valores de P e Q utilizados em cada critério.

\begin{tabular}{|l|c|c|}
\hline \multicolumn{1}{|c|}{ Critério } & P & Q \\
\hline Levantamento das necessidades & 10 & 5 \\
\hline Ambiente de Suporte & 20 & 10 \\
\hline Complexidade de implementação & 10 & 3 \\
\hline Avaliação das reações & 10 & 5 \\
\hline Impacto do treinamento no trabalho & 12 & 3 \\
\hline Resultado & 14 & 4 \\
\hline
\end{tabular}

Tabela 4: P e Q

Utilizou-se o método THOR para ordenação das alternativas. Os cálculos foram realizados mediante a utilização de um sistema computacional desenvolvido no Instituto Militar de Engenharia (IME). Os resultados encontrados nos cenários S1, S2 e S3 são apresentados na Figura 1.

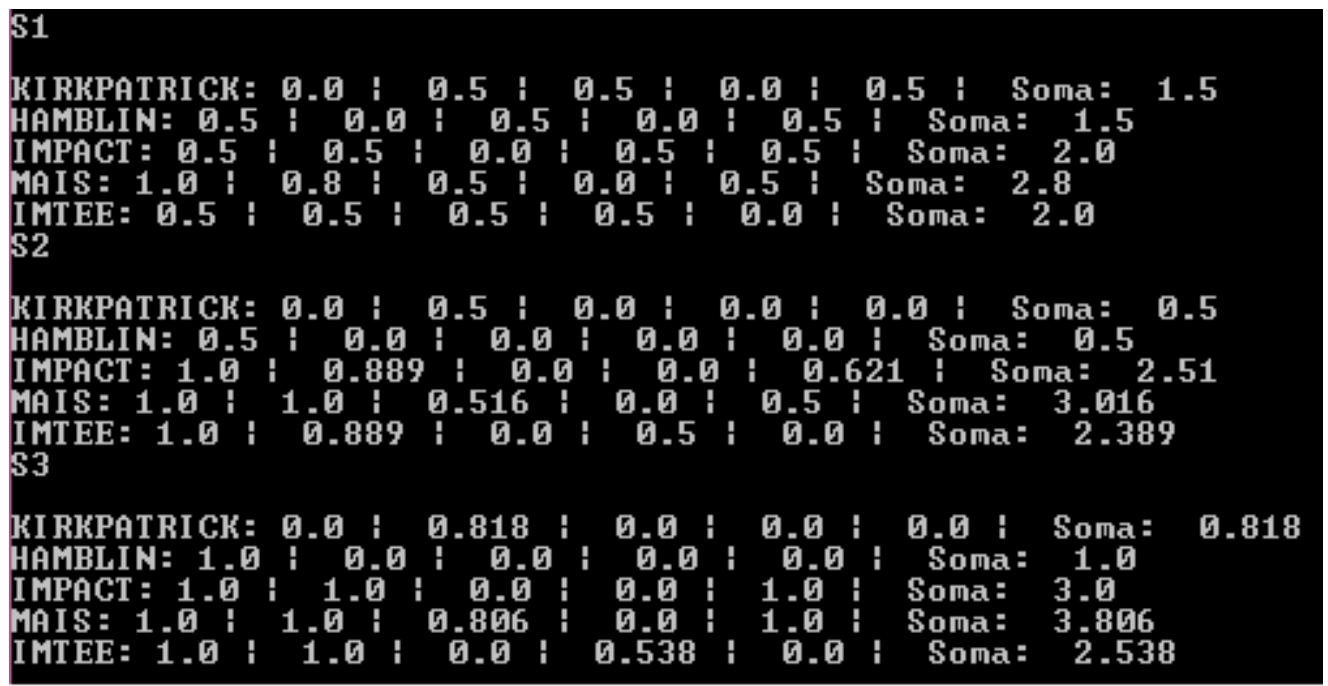

Figura 1: Tela do sistema computacional com a pontuação das alternativas em S1, S2 e S3

A partir dos resultados obtidos no software, chegou-se à seguinte ordenação das alternativas: 


\begin{tabular}{|c|c|c|c|c|}
\hline ORDENAÇÃO & ALTERNATIVAS & S1 & S2 & S3 \\
\hline $1^{\mathbf{o}}$ & MAIS & 2.8 & 3.016 & 3.806 \\
\hline $2^{\mathbf{o}}$ & IMPACT & 2.0 & 2.51 & 3.0 \\
\hline $3^{\mathbf{o}}$ & IMTEE & 2.0 & 2.389 & 2.538 \\
\hline $4^{\mathbf{o}}$ & HAMBLIN & 1.5 & 0.5 & 1.0 \\
\hline $5^{\mathbf{0}}$ & KIRKPATRICK & 0.0 & 0.5 & 0.5 \\
\hline
\end{tabular}

Tabela 5: Ordenação das alternativas

Sendo assim, após apresentação dos resultados para os decisores, chegou-se à conclusão de que o modelo MAIS foi escolhido como o modelo padrão que irá nortear o desenvolvimento das atividades de treinamento no Laboratório de Metrologia, por ser a alternativa com maior pontuação nas situações S1, S2 e S3.

As ações futuras propostas pelos facilitadores objetivam iniciar o processo de implementação do Modelo MAIS com a equipe responsável pela ministração dos treinamentos, promovendo ferramentas para a avaliação da eficácia.

\section{CONCLUSÃO E CONSIDERAÇÕES FINAIS}

Apesar do mercado ofertar capacitações em âmbito geral, há uma significativa lacuna entre a teoria destes treinamentos e a prática no cotidiano de um laboratório. Como solução, as empresas estão investindo na capacitação interna. O treinamento interno é uma ferramenta indispensável para que ocorram ganhos de conhecimento e habilidades, permitindo reduzir custos e tempo no processo de capacitação de equipes.

O Método THOR mostrou-se uma ferramenta adequada para balizar os processos de tomada de decisão em um laboratório de metrologia de pequeno porte, pois é possível chegar a um resultado objetivo.

Reflete-se também de que este método é perfeitamente adaptável ao tratamento de dados de diversos grupos, se mostrando uma excelente ferramenta para captar opiniões de diferentes usuários e gerar um resultado que compile essas percepções, pois já foi aplicado em diversas áreas de atuação com referido sucesso.

\section{REFERÊNCIAS BIBLIOGRÁFICAS}

[1] ABTD. O Panorama do treinamento no Brasil: Fatos, Indicadores, Tendências e Análises. São Paulo, 2017.

[2] NOVELLI, J. G. N. Avaliação em T\&D: por que mudar? T\&D, São Paulo, 1998.

[3] ABBAD, G. A.; LIMA, G. B. C.; VEIGA, M. R. M. Implantação de um sistema de avaliação de desempenho: métodos e estratégias. Revista de Administração, v. 31, n. 3, p. 38-52, 1996.

[4] ASSOCIAÇÃO BRASILEIRA DE NORMAS TÉCNICAS.NBR ISO/IEC 17025: Requisitos gerais para a competência de laboratórios de ensaios e calibração. Rio de Janeiro, 2017.

[5] GIL, A. C. Como Elaborar Projetos de Pesquisa. 4. ed. São Paulo: Atlas, 2002.

[6] LOUREIRO, R. O. As responsabilidades do treinamento. Acesso em: 04 de jun. de 2019. 
http://www.rh.com.br/Portal/Desenvolvimento/Artigo/2617/as-responsabilidades-dotreinamento.html >.

[7] FOLTRAN, C. U. et al. Treinamento e desenvolvimento de pessoas: o sucesso das organizações. Revista Alumni. São Paulo, 2012

[8] BORGES-ANDRADE, J. E. Avaliação somativa de sistemas instrucionais: Integração de três propostas. Tecnologia Educacional, 1982.

[9] CHIAVENATO, I. Recursos humanos. 7. ed. São Paulo: Atlas, 2002.

[10] BORGES-ANDRADE, J. E. Treinamento de pessoal: em busca de conhecimento e tecnologia relevantes para as organizações brasileiras. In: A. Tamayo, J. E. BorgesAndrade, \& W. Codo (Orgs.). Trabalho, Organizações e Cultura. (p.116-133). São Paulo: Cooperativa de Autores Associados, 1997.

[11] BORGES-ANDRADE, J. E. Desenvolvimento de medidas em avaliação de treinamento. Estudos de Psicologia (UFRN), v. 7, n. Especial, p. 31-43, 2002.

[12] SANTOS, M.; QUINTAL, R. S.; PAIXÃO, A. C.; GOMES, C. F. S. Simulation of Operation of an Integrated Information for Emergency Pre-hospital Care in Rio de Janeiro Municipality. Elsevier - Procedia Computer Science, v. 55, p. 931-938, 2015. DOI: 10.1016/j.procs.2015.07.111.

[13] ABBAD, G. Um modelo integrado de avaliação do impacto de treinamento no trabalho: IMPACT. Universidade de Brasília. Brasília, 1999.

[14] KIRKPATRICK, D. L. Evaluation of training: Training and development handbook. New York: McGraw Hill, 1976.

[15] HAMBLIN, A. C. Avaliação e controle do treinamento. São Paulo: McGraw-Hill do Brasil, 1978.

[16] TORRES, N. T.; GOMES, C. F. S. Metodologia Integrada para Tomada de Decisão com o Uso do Apoio Multicritério e a Análise Envoltória de Dados (Dea). In: Xl Simpósio Brasileiro de Pesquisa Operacional. João Pessoa - PB: Sociedade Brasileira de Pesquisa Operacional, 2008. p. 239-250.

[17] ABBAD, G., PILATI, R., BORGES-ANDRADE, J. E., \& SALlORENZO, L. H. Impacto do treinamento no trabalho: medida em amplitude. Artmed, Porto Alegre, 2012.

[18] ALVAREZ, K.; SALAS, E.; GAROFANO, C.M. An integrated model of training evaluation and effectiveness. Human Resource Development Review, Texas, 2004.

[19] BUCHANAN, John; GARDINER, Lorraine. (2003) A comparation of two reference point methods in multiple objective mathematical programmingg, EJOR, 149, pages 17-34.

[20] MATSATSINIS, N. F.; SAMARAS, A. P. (2001) MCDA and preferences disagregation in group decision support systems, European Journal of Operational Research 130, pag 414 a 429.

[21] LYRIO, M V. L.; ENSSLIN, L; DUTRA, A. Avaliação de desempenho em organizações públicas: análise de uma amostragem de publicações acadêmicas nas áreas de administração e contabilidade de 2002 a 2006. Congresso Brasileiro de Custos $-2007$.

[22] SANTOS, M. et al. Proposta de otimização do mix de produção utilizando o Método Simplex: um estudo de caso de uma confecção de moda íntima do município de 
Cordeiro - RJ. In: Anais do IV Simpósio de Engenharia de Produção: Lean Cost Management como filosofia global de otimização em organizações. Recife (PE): FBV, 2016.

[23] RANGEL, L. A. D.; GOMES, L. F. A. M. O Apoio Multicritério à Decisão na avaliação de candidatos. Production, Associação Brasileira de Engenharia de Produção, v. 20, n. 1, p. 92-101, 2010.

[24] GOMES, C. F. S. et al. Multicriteria decision making applied to waste recycling in Brazil. Omega, Pergamon, v. 36, n. 3, p. 395-404, jun 2008. ISSN 0305-0483.

[25] VINCKE, P. Multicriteria decision-aid. Chichester: John Wiley \& Sons, 1992.

[26] GOMES, C. F. S.; MAIA, A. C. C. Ordenação de alternativas de biomassa utilizando o apoio multicritério _a decisão. Production, Associação Brasileira de Engenharia de Produção, v. 23, n. 3, p. 488-499, 2013.

[27] XAVIER, L. H. et al. Multiple criteria decision making and environmental management through thor system assessment: Plastic material waste destination and performance evaluation of brazilian construction and demolition waste recycling facilities - case studies. In: XXXVI Simpósio Brasileiro de Pesquisa Operacional. São João del Rei - MG: Sociedade Brasileira de Pesquisa Operacional, 2004. Acesso em 20 de outubro de 2018.2 Disponível em: <https://www.researchgate.net/publication/264974465>.

[28] GOMES, C. F. S.; COSTA, H. G. Aplicação de métodos multicritério ao problema de escolha de modelos de pagamento eletrônico por cartão de crédito. Production, v. 25, n. 1, p. 54-68, 2015.

[29] ALENCAR, L. H.; COSTA, A. P. C. S.; GOMES, C. F. S. Gerenciamento de projeto na construção civil utilizando o apoio multicritério a decisão. Revista de la Escuela de Perfeccionamiento en Investigacion Operativa, v. 26, p. 163-186, 2005.

[30] GOMES, C. F. S. THOR - Um Algoritmo Híbrido de Apoio Multicritério à Decisão para Processos Decisórios com Alternativas Discretas. Tese (Tese de doutorado) | Universidade Federal do Rio de Janeiro, Rio de Janeiro, 1999.

[31] TENORIO, F. M.; SANTOS, M.; GOMES, C. F. S. Revisitando o método THOR: uma pesquisa bibliométrica. In: SIMPÓSIO DE PESQUISA OPERACIONAL E LOGÍSTICA DA MARINHA, 19., 2019, Rio de Janeiro, RJ. Anais [...]. Rio de Janeiro: Centro de Análises de Sistemas Navais, 2019.

As Referências Bibliográficas devem seguir as normas da ABNT/NBR 6023. 